\title{
Viscous properties and structure of ferro-fluids and magneto- rheological fluids. Non-equilibrium molecular dynamics (NEMD) studies of simple model systems
}

\author{
Journal Article \\ Author(s): \\ Hess, Siegfried; Weider, Titus; Kröger, Martin (D) \\ Publication date: \\ 2001-09 \\ Permanent link: \\ https://doi.org/10.3929/ethz-a-010678041
}

Rights / license:

In Copyright - Non-Commercial Use Permitted

Originally published in:

Magnetohydrodynamics 37(3) 


\title{
Viscous properties and structure of ferro-fluids and magneto-rheological fluids. Non-equilibrium molecular dynamics (NEMD) studies of simple model systems
}

\author{
S. Hess ${ }^{1}$, T. Weider ${ }^{1}$, M. Kröger ${ }^{1,2}$ \\ ${ }^{1}$ Institut f. Theoretische Physik, TU Berlin, Hardenbergstr. 36, 10623 Berlin, Germany \\ ${ }^{2}$ Polymerphysik, Institut f. Polymere, ETH Zürich, Sonneggstr. 3, 8092 Zürich, Schweiz
}

\begin{abstract}
A simple model is introduced for the theoretical treatment of ferro-fluids and magnetorheological (MR) fluids. Results from analytic calculations and from non-equilibrium molecular dynamics (NEMD) computer simulations are presented for the anisotropy of the viscosity and for shear-induced structural changes in fluids containing particles with perfectly oriented magnetic moments. Furthermore, the rheological behavior of fluids containing chains of particles is discussed.
\end{abstract}

Introduction In the presence of an external magnetic field, ferro-fluids show an anisotropic flow behavior. Phenomenologically, the anisotropy of the viscosity can be characterized by the same set of viscosity coefficients [1] as used for nematic liquid crystals in the presence of an orienting (electric or magnetic) field [2-7]. The microcopic mechanisms leading to the anisotropic viscous behavior, however, are rather different in these systems. And even for ferro-fluids, the microscopic mechanisms reponsible for the peculiar flow behavior of dilute solutions, where the magnetic-field-induced change of the viscosity is linear in the concentration of the ferro-fluid particles, is different from that of more concentrated solutions. There the direct anisotropic interaction between the ferro-fluid particles becomes the dominating mechanism. Theoretical descriptions applicable to the dilute solution are given in $[8,9]$. To highlight the phenomena associated with the second case, a simple model is studied here. It has previously been used to treat equililibrium [10] and non-equilibrium properties [10-14] of dense ferro-fluids and of MR fluids, where a yield stress can occur in the presence of a magnetic field. Chains break down under shear. As a consequence, one observes a strong shear thinning. This not only occurs in MR fluids, but also in ferro-fluids composed of particles with permanent magnetic dipole moments, even without an applied field. Methods developped for the study of flow properties of 'living polymers' [15-17] and actin filaments [18] can be applied to ferro-fluids.

\section{Description of the viscosity}

1.1. General remarks, isotropic liquids The viscosity characterizes the transport of linear momentum through a fluid. For a simple geometry where the flow velocity $\mathbf{v}=\left(v_{x}(y), 0,0\right)$ is in the $x$-direction and where $\partial v_{x} / \partial y$ is the only nonvanishing component of the velocity gradient tensor, the (shear) viscosity is defined as the ratio of the component $p_{y x}$ of the pressure tensor and shear rate $\Gamma=\partial v_{x} / \partial y$ through $p_{y x}=-\eta \Gamma$. For a more general geometry, a tensorial description is needed. Here, cartesian components are denoted by Greek subscripts. The summation convention is used. The pressure tensor $P_{\nu \mu}$ occuring in the local conservation equation for the linear momentum $\rho d v_{\mu} / d t+\nabla_{\nu} P_{\nu \mu}=0$, is the sum of its equilibrium value $P_{\mathrm{eq}} \delta_{\nu \mu}$, where $\delta_{\nu \mu}$ is the unit tensor, and of the friction 
pressure tensor $p_{\nu \mu}: P_{\nu \mu}=P_{\mathrm{eq}} \delta_{\nu \mu}+p_{\nu \mu}$. Like any second rank tensor, the latter quantity can be decomposed into its isotropic, antisymmetric and symmetric traceless parts:

$$
p_{\nu \mu}=p \delta_{\nu \mu}+\frac{1}{2} \epsilon_{\nu \mu \kappa} p_{\kappa}^{\mathrm{a}}+\widetilde{p_{\nu \mu}},
$$

where $p=(1 / 3) p_{\lambda \lambda}$ is one third of the trace of the friction pressure tensor. The antisymmetric part of the tensor involves the pseudo vector $p_{\lambda}^{\mathrm{a}}=\epsilon_{\lambda \nu \mu} p_{\nu \mu}$, the quantity $\epsilon \ldots$ is the totally antisymmetric isotropic third rank tensor. With the help of this tensor, the cross product of two vectors $\mathbf{a}$ and $\mathbf{b}$ is given by $(\mathbf{a} \times \mathbf{b})_{\lambda}=$ $\epsilon_{\lambda \nu \mu} a_{\nu} b_{\mu}$. The symbol $\ldots$ indicates the symmetric traceless part of a tensor, in particular: $\overline{p_{\nu \mu}}=\left(p_{\nu \mu}+p_{\mu \nu}\right) / 2-p_{\lambda \lambda} \delta_{\nu \mu} / 3$. The velocity gradient tensor can be decomposed, in analogy to (1), $\nabla_{\nu} v_{\mu}=\frac{1}{3} \nabla_{\lambda} v_{\lambda} \delta_{\nu \mu}+\epsilon_{\nu \mu \kappa} \omega_{\kappa}+\gamma_{\nu \mu}$. The divergence $\nabla_{\lambda} v_{\lambda}$ of the velocity field vanishes for a volume conserving flow of a practically incompressible fluid. The quantity $\underline{\omega}=(1 / 2) \underline{\nabla} \times \mathbf{v}$ is the vorticity of the flow field. The symmetric traceless part of the velocity gradient tensor, $\gamma_{\nu \mu}=\overline{\nabla_{\nu} v_{\mu}}=\left(\nabla_{\nu} v_{\mu}+\nabla_{\mu} v_{\nu}\right) / 2-\nabla_{\lambda} v_{\lambda} \delta_{\nu \mu} / 3$, is referred to as the deformation rate tensor.

The antisymmetric part of the pressure tensor is identically zero for substances composed of spherical particles without any internal rotational degree of freedom. Under most practical circumstances, in particular for stationary situations and in the absence of external fields, the antisymmetric part of the pressure tensor becomes zero for molecular fluids in the isotropic phase. As a consequence, the quantity $\mathbf{p}^{a}$ vanishes. Then, in the linear flow regime, two viscosity coefficients, viz. the above mentioned shear viscosity $\eta$ and the bulk or volume viscosity $\eta_{\mathrm{V}}$, suffice to characterize the viscous behavior of an isotropic fluid: $\overline{p_{\nu \mu}}=-2 \eta \bar{\nabla}_{\nu} v_{\mu}, p=$ $-\eta_{\mathrm{V}} \underline{\nabla} \cdot \mathbf{v}$. Note again that for an incompressible fluid, or for a volume conserving flow, one has $\underline{\nabla} \cdot \mathbf{v}=0$.

1.2. The viscosity coefficients of nematic liquid crystals and of ferro-fluids For a nematic liquid crystal with the director field $\mathbf{n}=\mathbf{n}(t, \mathbf{r})$, and with the corotational time derivative of the director denoted by $\mathbf{N}: N_{\mu}=d n_{\mu} / d t-\varepsilon_{\mu \nu \lambda} \omega_{\nu} n_{\lambda}$ the ansatz $[1,5]$

$$
\begin{aligned}
-p_{\nu \mu}= & \alpha_{1} n_{\nu} n_{\mu} n_{\lambda} n_{\kappa} \gamma_{\lambda \kappa}+\alpha_{2} n_{\nu} N_{\mu}+\alpha_{3} n_{\mu} N_{\nu}+\alpha_{4} \gamma_{\nu \mu}+\alpha_{5} n_{\nu} n_{\lambda} \gamma_{\lambda \mu} \\
& +\alpha_{6} n_{\mu} n_{\lambda} \gamma_{\lambda \nu}+\zeta_{1} n_{\lambda} n_{\kappa} \gamma_{\lambda \kappa} \delta_{\mu \nu}+\zeta_{2} n_{\nu} n_{\mu} \nabla_{\lambda} v_{\lambda}+\zeta_{3} \nabla_{\lambda} v_{\lambda} \delta_{\mu \nu}
\end{aligned}
$$

is used to describe the viscosity. This relation is linear in the velocity gradient and in the corotational derivative of the director. Furthermore, it is invariant under the replacement of $\mathbf{n}$ by $\mathbf{- n}$, as it should. The Leslie coefficients $\alpha_{1}, \ldots, \alpha_{6}$ and the coefficients $\zeta_{1}, \zeta_{2}, \zeta_{3}$ have the dimension of a viscosity.

Decomposition of the pressure tensor and of the velocity gradient tensor into their isotropic, antisymmetric and symmetric traceless parts leads to the linear constitutive laws [1]

$$
\begin{aligned}
\overline{p_{\nu \mu}}= & -2 \eta \gamma_{\nu \mu}-2 \tilde{\eta}_{1} \overline{n_{\nu} n_{\lambda}} \gamma_{\lambda \mu}-2 \tilde{\eta}_{2} \overparen{n_{\nu} N_{\mu}} \\
& -2 \tilde{\eta}_{3} \overline{n_{\nu} n_{\mu}} n_{\lambda} n_{\kappa} \gamma_{\lambda \kappa}-\zeta_{2} \overline{n_{\nu} n_{\mu}} \nabla_{\lambda} v_{\lambda} \\
p_{\lambda}^{a}= & \epsilon_{\lambda \nu \mu}\left(\gamma_{1} n_{\nu} N_{\mu}+\gamma_{2} \overline{n_{\nu} n_{\sigma}} \gamma_{\sigma \mu}\right) \\
\frac{1}{3} p_{\lambda \lambda}= & -\eta_{\mathrm{V}} \nabla_{\lambda} v_{\lambda}-\kappa n_{\lambda} n_{\kappa} \gamma_{\lambda \kappa} .
\end{aligned}
$$

The viscosity coefficients used in Eqs. 3-5 are related to those of Eq. 2 by $2 \eta=\alpha_{4}+\left(\alpha_{5}+\alpha_{6}\right) / 3,2 \tilde{\eta}_{1}=\left(\alpha_{5}+\alpha_{6}\right), 2 \tilde{\eta}_{2}=\left(\alpha_{2}+\alpha_{3}\right), 2 \tilde{\eta}_{3}=\alpha_{1}, \gamma_{1}=\alpha_{3}-\alpha_{2}$, 
$\gamma_{2}=\alpha_{6}-\alpha_{5}, \eta_{\mathrm{V}}=\zeta_{2} / 3+\zeta_{3}$, and $\kappa=\zeta_{1}+\left(\alpha_{1}+\alpha_{5}+\alpha_{6}\right) / 3$. The dyadic $\overline{\mathbf{n n}}$ stems from the second rank alignment tensor. The coefficients linking second with first rank tensors, and second rank tensors with scalars (rank 0), obey Onsager symmetry relations. These are $2 \tilde{\eta}_{2}=\gamma_{2}, \zeta_{2}=\kappa$, where the first of these relations is equivalent to the Onsager-Parodi relation [4] $\alpha_{2}+\alpha_{3}=\alpha_{6}-\alpha_{5}$. Due to the symmetry relations, only 7 of the 9 viscosity coefficients are linearly independent. In an isotropic fluid all coefficients vanish except for the shear viscosity $\eta=\frac{1}{2} \alpha_{4}$ and the bulk viscosity $\eta_{\mathrm{V}}=\zeta_{3}$. Positive entropy production requires $\eta>0$, $\eta_{\mathrm{V}}>0, \gamma_{1}>0$ but $\tilde{\eta}_{1}, \tilde{\eta}_{2}, \tilde{\eta}_{3}$ and $\gamma_{2}$ may have either sign. One standard set of viscosity coefficients are the $\alpha_{1}, \ldots, \alpha_{6}$ of Eq. 2 . The coefficients occurring in the ansatz (3-5) can be preferable in connection with theoretical considerations. In experiments and in NEMD simulations, linear combinations of the 'basic' viscosity coefficients are measured and calculated. Some examples are mentioned next.

In a plane Couette or a plane Poiseuille flow with the velocity in the $x$ direction and its gradient in the $y$-direction one has $\nabla_{\nu} v_{\mu}=\Gamma e_{\nu}^{y} e_{\mu}^{x}, \omega_{\mu}=-\Gamma e_{\mu}^{z} / 2$, with the shear rate $\Gamma=\partial v_{x} / \partial y$, and $\mathbf{e}^{x, y, z}$ are unit vectors parallel to the $x_{-}, y^{-}, z-$ coordinate axes.

The Miesowicz viscosities $\eta_{i}, i=1,2,3$ are defined as the ratio of the negative $y x$-component of the pressure tensor and the shear rate $\Gamma: p_{y x}^{(i)}=-\eta_{i} \Gamma$. The label $i=1,2,3$ refers to the cases where the director $\mathbf{n}$ is parallel to the $x$-, $y$ ,$z$-axis, respectively. The orienting (magnetic) field has to be strong enough to overcome the flow induced orientation. A fourth coefficient $\eta_{4}$ with $\mathbf{n}$ parallel to the bisector between the $x$ - and $y$-axes is needed to characterize the shear viscosity completely. Instead of $\eta_{4}$, the Helfrich viscosity coefficient $\eta_{12}=4 \eta_{4}-2\left(\eta_{1}+\eta_{2}\right)$ is used in addition to the Miesowicz coefficients. The 'rotational' viscosity $\gamma_{1}$ can be measured via the torque exerted on a nematic liquid crystal in the presence of a rotating magnetic field: Tsvetkov effect [19-21].

The four effective viscosities measurable in a flow experiment are related to the viscosity coefficients of Eq. 2 and Eqs. 3-5 by

$$
\begin{aligned}
& \eta_{1}=\frac{1}{2}\left(\alpha_{4}+\alpha_{6}+\alpha_{3}\right)=\eta+\frac{1}{6} \tilde{\eta}_{1}+\frac{1}{2} \tilde{\eta}_{2}+\frac{1}{4}\left(\gamma_{1}+\gamma_{2}\right), \\
& \eta_{2}=\frac{1}{2}\left(\alpha_{4}+\alpha_{5}-\alpha_{2}\right)=\eta+\frac{1}{6} \tilde{\eta}_{1}-\frac{1}{2} \tilde{\eta}_{2}+\frac{1}{4}\left(\gamma_{1}-\gamma_{2}\right), \\
& \eta_{3}=\frac{1}{2} \alpha_{4}=\eta-\frac{1}{3} \tilde{\eta}_{1}, \eta_{12}=4 \eta_{4}-2\left(\eta_{1}+\eta_{2}\right)=\alpha_{1}=2 \tilde{\eta}_{3} .
\end{aligned}
$$

The antisymmetric part of the pressure tensor contributes to $\eta_{1}$ and $\eta_{2}$ but not to $\eta_{3}$ and $\eta_{12}$. Notice that $\eta_{1}+\eta_{2}+\eta_{3}=3 \eta+\gamma_{1} / 2, \eta_{1}-\eta_{2}=2 \tilde{\eta}_{2}=\gamma_{2}$, $\eta_{1}+\eta_{2}-2 \eta_{3}=\tilde{\eta}_{1}$. By symmetry arguments, the same set of vicsosity coefficients can be used for ferro-fluids in the presence of a magnetic field. The director has to be replaced by a unit vector parallel to the local magnetization which, for strong applied fields, is parallel to the direction of the external field. The following considerations are limited to the case of either zero or a 'strong' field.

Theories appropriate for dilute solutions which just take into account that the magnetic field affects or hinders the rotation of a particle and associate the magneto-viscous effect solely with the antisymmetric part of the pressure tensor, imply that $\gamma_{1}$ is nonzero and depends on the field strength and the shear rate. In that case, however, one has $\tilde{\eta}_{1}=0, \tilde{\eta}_{2}=0$, and consequently $\eta_{1}-\eta_{2}=$ $\gamma_{2}=0$, as well as $\eta_{12}=0$. Then the McTague [22] viscosity coefficients $\eta_{\|}=$ $\eta_{1}$ and $\eta_{\perp}=\left(\eta_{2}+\eta_{3}\right) / 2$ become equal to $\eta+\gamma_{1} / 4$ and $\eta+\gamma_{1} / 8$, respectively. The anisotropy observed for the ferro-fluids of [22] are indeed of that type. In general, the anisotropy found in more concentrated ferro-fluids is more complex 
and it is expected that all viscosity coefficients needed by symmetry considerations will be nonzero. So far, there is no precise experimental data available for the complete set of coefficients. However, experimentally observed effects due to the influence of magnetic fields on the rotation of single magnetic nanoparticles as well as cooperative phenomena and their importance for viscous effects in ferrofluids have been reviewed in $[23,24]$. In particular, a change of field-induced increase of viscosity due to variation of the shear rate applied to the fluid has been reported in [25], see also Refs. [26]. The transition area between elastic and viscous behavior for a conventional ER fluid and a state-of-the-art MR fluid through the use of oscillatory rheometry techniques has been investigated [27]. More recently, neutron scattering [28] and microgravity [29] have been applied to resolve structural effects and flow profiles in MR fluids. Inhomogeneities in particle concentration have been considered in order to explain an enormeous increase of yield stresses of ER and MR suspensions subjected to electric or magnetic fields in $[30]$.

2. Basics of molecular dynamics In a molecular dynamics (MD) computer simulation for a substance composed of $N$ particles, Newton's equations of motion $m d^{2} / d t^{2} \mathbf{r}^{i}=\mathbf{F}^{i}=\sum_{j} \mathbf{F}^{i j}$ are integrated numerically. The particles are located at positions $\mathbf{r}^{i}$ in a volume $V$. The particle density is $n=N / V$. The particle $i,(i=1,2, \ldots, N)$, feels the force $\mathbf{F}^{i}=\sum_{j} \mathbf{F}^{i j}$, which is the sum of the forces $\mathbf{F}^{i j}$ exerted by all other particles $j \neq i$ on particle $i$. When one wants to avoid surface effects, periodic boundary conditions and the nearest image convention are used. This means particle $i$ either feels the force caused by particle $j$ or by one of its images depending on which one is closest to it. For particles with rotational degrees of freedom, also equations of motion for angle variables, involving the torque acting on particles, have to be solved.

The temperature $T$ of the system is linked with the part of the kinetic energy $K$ which is not associated with a macroscopic motion: $3(N-1) k_{\mathrm{B}} T / 2=K:=$ $\sum_{i} m\left(\mathbf{c}^{i}\right)^{2} / 2$, where $k_{\mathrm{B}}$ is Boltzmann's constant and $\mathbf{c}^{i}$ is the 'peculiar velocity', i.e. the velocity of a particle relative to the flow velocity $\mathbf{v}=\mathbf{v}\left(\mathbf{r}^{\mathbf{i}}\right)$. In order to simulate an isothermal system the temperature has to be kept constant. The simplest version of a 'thermostat' involves rescaling the peculiar velocity after each time step by the factor $\left(T_{\text {wanted }} / T_{\text {measured }}\right)^{1 / 2}$. Other thermostats, e.g. those referred to as 'Gaussian' and 'Nosé-Hoover' [31], are imposed as constraints.

The observables of interest, such as the internal energy and the components of the pressure or the stress tensor, can be calculated from the known positions and velocities of the particles as time averages according to the rules of statistical physics. Similarly, more detailed information can be obtained from the simulation, such as the velocity distribution function, the pair correlation function or the static structure factor, which can also be measured in scattering experiments. In NEMD simulations, relaxation and transport phenomena are investigated directly and in close analogy to real experiments [12].

Dimensionless or 'scaled' variables are used which are denoted by the same symbols as the physical variables when no danger of confusion exists. For a system of spherical particles whose forces are derived from Lennard-Jones (LJ) potential $\Phi$ depending on the distance $r: \Phi=\Phi^{L J} \equiv 4 \Phi_{0}\left[\left(r_{0} / r\right)^{12}-\left(r_{0} / r\right)^{6}\right]$, lengths and energies are presented in units of the diameter $r_{0}$ and of the potential depth $\Phi_{0}$. The units used for the particle density and for the temperature are $r_{0}^{-3}$ and $k_{\mathrm{B}}^{-1} \Phi_{0}$. The time is scaled with the reference time $t_{0}=r_{0} m^{1 / 2} \Phi_{0}^{-1 / 2}, m$ is the mass of a particle. The pressure, the shear rate and the viscosity of the LJ-fluid are expressed in units of $r_{0}^{-3} \Phi_{0}, t_{0}^{-1}$ and $r_{0}^{-3} \Phi_{0} t_{0}=r_{0}^{-2} m^{1 / 2} \Phi_{0}^{1 / 2}$. In the simulations, the cut- 
off of the interaction at a finite distance $r_{\text {cut }}$ is often achieved just by putting the potential and the force equal to zero for $r>r_{\text {cut }}$, e.g. with $r_{\text {cut }}=2.5 r_{0}$.

When only the repulsive $r^{-12}$-part of the LJ interaction potential is taken into account, one speaks of a 'soft spheres' (SS) potential. Often, this potential is written as $\Phi=\Phi_{0}^{S S}\left(r_{0} / r\right)^{12}$ without the factor 4 of the LJ potential. When reduced SS-units with $\Phi_{0}^{S S}$ as energy reference are used, $T=1 / 4$ in SS-units is equivalent to $T=1$ in LJ-units. Results to be reported in the following have been obtained for this particular temperature $T=1 / 4$.

2.1. Plane Couette flow, pressure tensor and viscosity A simple shear flow in the $x$-direction with the gradient in the $y$-direction can be generated either by moving boundaries or forces [32-34], or as implemented here, by moving image particles undergoing an ideal Couette flow with the prescribed shear rate $\Gamma=$ $\partial v_{x} / \partial y$ (homogeneous shear). For details on the realization of a NEMD shear flow we wish to refer to the reviews $[12,13]$ and references cited herein.

Rheological properties such as the (non-newtonian) viscosity and the normal pressure differences are obtained from the cartesian components of the stress tensor $\sigma_{\mu \nu}=-p_{\mu \nu}$ or of the pressure tensor $p_{\mu \nu}$ which is the sum of 'kinetic' und 'potential' contributions: $p_{\mu \nu}=p_{\mu \nu}^{\mathrm{kin}}+p_{\mu \nu}^{\mathrm{pot}}, V p_{\mu \nu}^{\mathrm{kin}}=\sum_{i} m_{i} c_{\mu}^{i} c_{\nu}^{i}$, and $V p_{\mu \nu}^{\mathrm{pot}}=$ $\frac{1}{2} \sum_{i j} r_{\mu}^{i j} F_{\nu}^{i j}$. Here $\mathbf{c}^{i}$ is the peculiar velocity of particle $i$, i.e. its velocity relative to the flow velocity $\mathbf{v}\left(\mathbf{r}^{i}\right), \mathbf{r}^{i j}=\mathbf{r}^{i}-\mathbf{r}^{j}$ is the relative position vector of particles $i, j$ and $\mathbf{F}^{i j}$ is the force acting between them. As before, the Greek subscripts $\mu, \nu$, which assume the values $1,2,3$, stand for cartesian components associated with the $x, y, z$-directions. In the simulations, the expression for the pressure tensor given is averaged over many $\left(10^{3}\right.$ to $\left.10^{6}\right)$ time steps. For the present flow geometry, the (non-newtonian) viscosity $\eta$ is obtained by dividing the $y x(21)$-component of the stress or pressure tensor by the shear rate: $\eta=\sigma_{y x} / \Gamma=-p_{y x} / \Gamma$.

From the simulation, the kinetic and potential contributions to the pressure tensor and to the viscosity can be computed separately. Only the sum can be measured in a real experiment. The kinetic contribution to the viscosity dominates in dilute gases [35]. In dense fluids (liquids) the potential contribution is more important. The same applies to colloidal dispersion, here treated as 'macro fluids'.

\section{Ferro-fluids and magneto-rheological fluids}

3.1. The model The model studied here deals with relatively dense solutions of ferro-fluid particles which, typically, occupy 10 per cent or more of the available volume. It is assumed that the applied magnetic field is strong enough, such that all magnetic moments are perfectly parallel to the external field. In addition to a repulsive isotropic interaction, the angle dependence of the dipole-dipole interaction is taken into account. For computational simplicity, the interaction is cut-off at a finite distance. Thus effects associated with the long range character of the dipole-dipole interaction are disregared. Furthermore, it is assumed that the dynamics is dominated by the particle-particle interaction, the back ground fluid (solvent) enters only via the local thermostat which removes the heat generated in a viscous flow. Of course, additional friction forces and fluctuacting forces due to the solvent can and have been taken into account [36].

Spherical colloidal particles with a magnetic core as they occurr in ferro-fluids in the presence of an applied magnetic field parallel to the unit vector $\mathbf{n}$ have been modelled by soft spheres plus a dipole-dipole interaction (SSD) [37]:

$$
\Phi=\Phi_{0}^{\mathrm{SS}}\left[\left(\frac{r_{0}}{r}\right)^{12}-\epsilon_{\mathrm{mag}}\left(\frac{r_{0}}{r}\right)^{3}\left(r^{-2}(\mathbf{r} \cdot \mathbf{n})^{2}-\frac{1}{3}\right)\right] .
$$




\begin{tabular}{lccccccc}
\hline \hline model & $\eta_{1}$ & $\eta_{2}$ & $\eta_{3}$ & $\eta_{12}$ & $\gamma_{1}$ & $\gamma_{2}$ & $\kappa$ \\
\hline SSD & $0.63(5)$ & $0.43(4)$ & $0.50(5)$ & $2.0(1)$ & $0.16(2)$ & $0.17(2)$ & $0.6(1)$ \\
\hline \hline
\end{tabular}

Table 1: The (potential contributions to the) Miesowicz viscosities $\eta_{1}, \eta_{2}, \eta_{3}$, the Helfrich viscosity $\eta_{12}$, the Leslie viscosities $\gamma_{1}, \gamma_{2}$ and the coupling coefficient $\kappa$ of the SSD ferro-fluid at $n=0.6$, for $\epsilon_{\text {mag }}=2.4$. The data are inferred from the NEMD simulations, in the limit of small shear rates.

The parameter $\epsilon_{\mathrm{mag}}>0$ is proportional to the square of the (induced) magnetic moments of the particles which are parallel to $\mathbf{n}$. The quantities $r_{0}$ and $\Phi_{0}^{\mathrm{SS}}$ set the length and energy scales. The interaction is cut-off at $r_{\text {cut }}=2.5 r_{0}$.

3.2. Viscosity coefficients Pairs of particles feel a disc-like interaction since, for fixed relative kinetic energy, they can approach each other more closely in the direction parallel to $\mathbf{n}$ than in the perpendicular directions. Thus it is not surprising that ferro-fluids show an anisotropy analogous to nematic discotic liquid crystals $[1,38]$. In Tab. 1 , NEMD results are presented for the only state point where all viscosities (except the bulk viscosity) have been determined.

3.3. From ferro-fluids to magneto-rheological fluids When the dipole-dipole interaction is stronger, however, chains are formed which, at higher densities, are arranged in partially ordered spatial structures. This affects the viscous behavior in a dramatic way. An example is shown in Fig. 1 where the viscosities $\eta_{1}$ (magnetic field parallel to the flow velocity) and $\eta_{2}$ (magnetic field parallel to the gradient of the flow velocity) are plotted as functions of the anisotropy parameter $\epsilon_{\mathrm{mag}}$. The state point is $n=0.6$ in SS-units and the shear rate is $\Gamma=0.06$ (and $T=1 / 4$ of course). The interaction is cut-off at $r_{\text {cut }}=2.5 r_{0}$, and $\left(r_{0} / r\right)^{3}$ in (7) is replaced by $\left(r_{0} / r\right)^{3}-\left(r_{0} / r_{\text {cut }}\right)^{3}+3\left(r_{0} / r_{\text {cut }}\right)^{4}\left(r / r_{0}-r_{\text {cut }} / r_{0}\right)$ in order to achieve a smoother cutoff. The simulations were made with $N=1000$ particles [39]. For $0<\epsilon_{\text {mag }}<3$, the discotic behavior $\eta_{1}>\eta_{2}$ is seen in Fig. 1. For $\epsilon_{\text {mag }}>3$, the viscosity $\eta_{2}$ for the field parallel to the gradient direction increases strongly with increasing $\epsilon_{\text {mag }}$. Notice that a logarithmic scale is used for the viscosity.

The viscosities and the shear induced structural changes were analysed in the orientations 1 and 2, for number densities between $n=0.2$ and 0.8 , corresponding to packing fractions of about 0.1 to 0.4 , and for the magnetic interaction strength

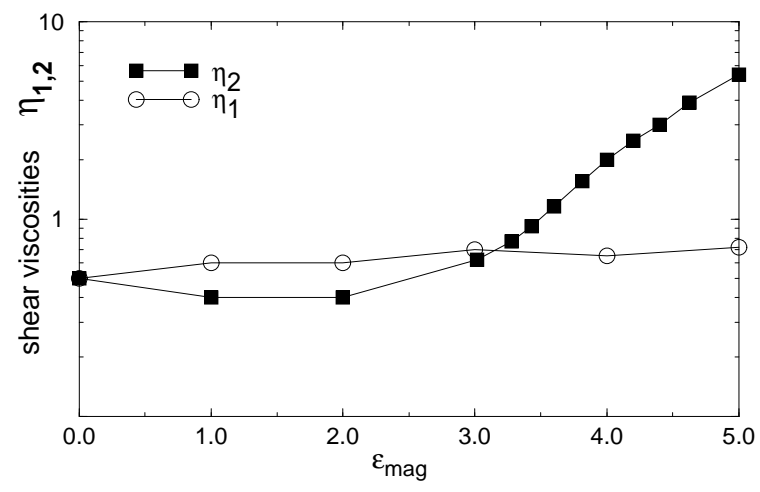

Figure 1: The viscosity coefficients $\eta_{1}, \eta_{2}$ as function of the strength $\epsilon_{\mathrm{mag}}$ of the dipole-dipole interaction for $n=0.6$ and $\Gamma=0.06$ in SS-units. 


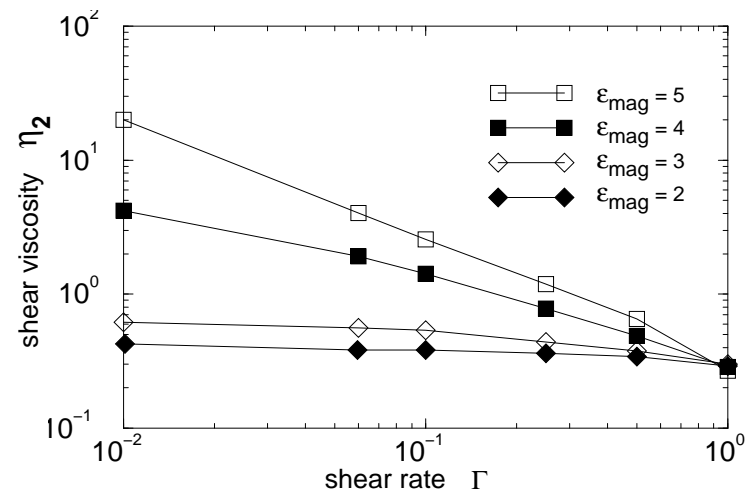

Figure 2: The effect of $\epsilon_{\text {mag }}$ on the potential contribution to the shear viscosity $\eta_{2}$ vs shear rate $\Gamma$ in SS units.

$\epsilon_{\text {mag }}$ ranging from 0 to 8 . Fig. 2 shows results for a specific case of interest. Breaking and reformation of chains and of more complex structures, under shear flow, have been observed.

A yield stress occurs for the higher values of the dipole-dipole interaction. This is typical for the MR fluids, which are similar to the ferro-fluids but which are composed of particles with stronger dipole-dipole interaction and usually contain a higher volume fraction of colloidal particles. Electro-rheological (ER) fluids can, with appropriate modifications, also be treated theoretically by the model. For simulations where solvent effects and hydrodynamic interactions are also taken into account, see [36]. The transition from the ferro-fluid to the magneto-rheological behavior, with increasing magnetic interaction, is analgous to what one might expect in a nematic discotic system which can undergo a transition to a columnar phase, at lower temperatures.

3.4. Shear thinning and shear-induced structural changes The chains formed in MR fluids break and are reformed under shear. The viscosity becomes smaller with increasing shear rate: shear thinning. In Fig. 2, the viscosity $\eta_{2}$, viz. with the magnetic field parallel to the gradient direction, is plotted as function of the shear rate, for various values of the parameter $\epsilon_{\text {mag }}$ determining the strength of the dipole-dipole interaction. The behavior seen is typical for MR fluids.

Structural changes associated with this behavior can and have been analysed in the simulations by inspecting snapshots of particle positions, by computing (partial) pair-correlation functions and the anisotropy of the static structure factor. the latter quantity can be measured, e.g. in neutron scattering experiments [28]. An example for the influence of an increasing shear rate on the structure factor is shown in Fig. 3.

4. Shear-induced breakdown of chains Ferro-fluids composed of particles with strong permanent dipole moments and sufficently high concentration form wormlike chains even when no magnetic field is applied [40]. The chains can break by thermal agitation, and even more so, when subjected to a shear flow. This leads to a pronounced shear thinning behavior, analogous to that seen in NEMD computer simulation studies of wormlike micellar systems, cf. [16]. Given the strength of the 'binding energy' or 'scission energy' $E_{\mathrm{sc}}$ between neighbors in a linear chain, the dependence of the average (dimensionless) chain length $\bar{L}$ on the shear rate can be computed [17]. In equilibrium, one expects - for stiff linear chains - a simple exponential increase of the number averaged length with $E_{\mathrm{sc}}$ 
a)

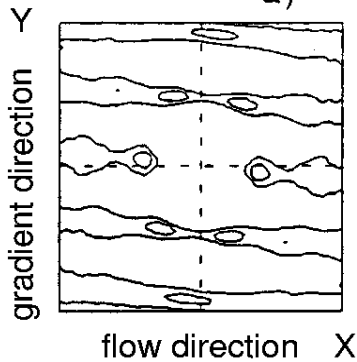

b)

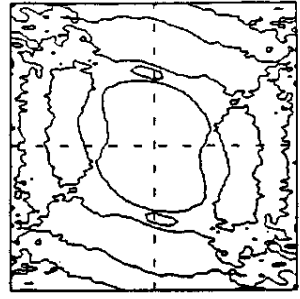

c)

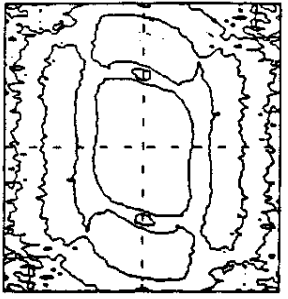

Figure 3: Effect of shear rate on the static structure factor $(n=0.6$, magnetic field in $y$-direction, magnetic interaction strength $\epsilon_{\text {mag }}=6$ ), contour-projected onto the shearing plane, extracted from NEMD particle configurations for three shear rates $\Gamma=0.01(\mathrm{a}), \Gamma=0.5(\mathrm{~b})$, and $\Gamma=1(\mathrm{c})$.

and a square root increase with concentration, i.e., $\bar{L}_{\mathrm{eq}} \propto \phi^{1 / 2} \exp \left(E_{\mathrm{sc}} / 2 k_{\mathrm{B}} T\right)$. For the MR system under consideration, the scission energy may be roughly estimated from the energy minimum in (7), with $\mathbf{r} \| \mathbf{n}$, i.e., $E_{\mathrm{sc}}=\Phi_{0} \epsilon_{\text {mag }}^{4 / 3} 48^{-1 / 3}$. We therefore expect for the number average: $\bar{L}_{\text {eq }} \propto \phi^{1 / 2} \exp \left(\epsilon_{\text {mag }}^{4 / 3} / 6^{1 / 3}\right)$, and a monoexponential distribution of lengths, at low concentrations. Corrections for higher concentrations and the effect of semiflexibility (persistence length) and ring formation on the shape of agglomerates has been discussed, e.g., in Ref. [41]. The effect of alignment and stretching of transient chains on their length distribution, the shear viscosity and normal stresses in the (magnet) field-free case has been estimated in the framework of a modified Rouse model [17], a sample result is reproduced in Fig. 4.

Due to the stretching and the alignment, shear thinning is also observed when chains do not break. From the computer simulations we infer that the shear rate dependence of the normal stress differences is a more sensitive indicator of breaking of chains. Thus measurements of at least the first normal pressure difference of ferro-fluids are of interest.

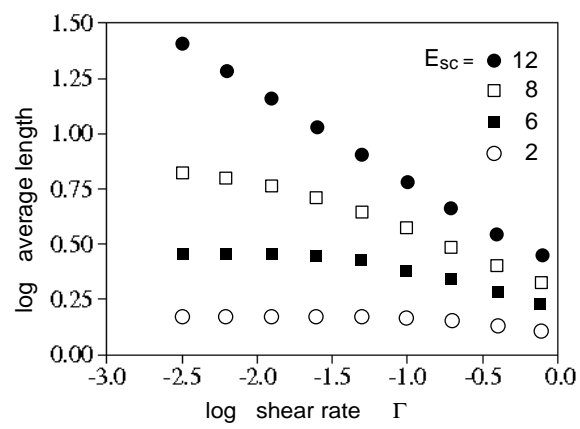

Figure 4: Analytically predicted effect of shear rate on the average length $\bar{L}$ for a wormlike micellar solution ( $4 \%$ concentration) parametrized in the scission energy $E_{\mathrm{sc}}$ [17]. A relationship between the behavior of MR fluids and wormlike micellar solutions is established in the text. 


\section{REFERENCES}

1. S. Hess. J. Non-Equilib. Thermodyn 11 (1986) 175.

2. M. Miesowicz. Nature 17 (1935) 261; 158 (1946) 27.

3. J.L. Ericksen. Arch. Ratl. Mech. Anal. 4 (1960) 231; Mol. Cryst. Liq. Cryst. 7 (1969) 153; F.M. Leslie, J. Mech. Appl. Math. 19 (1966) 357; Arch. Ratl. Mech. Anal. 28 (1968) 265.

4. O. Parodi. J. Phys. 31 (1970) 581.

5. P.G. De Gennes. The Physics of Liquid Crystals (Clarendon Press, Oxford, 1974), Chap. 3.

6. H. Kelker ANd R. HATz. Handbook of Liquid Crystals (Verlag Chemie, Weinheim, 1980)

7. G. Vertogen And W. DeJeu. Thermotropic Liquid Crystals, Fundamentals, Chem. Phys. 45 (Springer, Berlin, 1988)

8. M.I. Shliomis. Sov. Phys. 34 (1972) 1291.

9. W.F. Hall and S.N. Busenberg. J. Chem. Phys. 51 (1969) 137.

10. S. Hess, J.B. Hayter, And R. Pynn. Molec. Phys. 53 (1984) 1527; S. Hess. Structure and dynamics of oriented ferrofluids, in: Physics of Complex and Supermolecular Fluids, eds. S.A. Safran and N.A. Clark (J. Wiley, New York 1987) p. 631.

11. S. Hess, J.F. Schwarzl And D. BaAlss. J. Phys. Condens. Mat. 2 8A (1990) 279.

12. S. Hess, M. Kröger, W. Loose, C. Pereira Borgmeyer, R. Schramek, H. Voigt, And T. Weider. Simple and Complex Fluids under Shear, in: Monte Carlo and Molecular Dynamics of Condensed Matter Systems, eds. K. Binder and G. Ciccotti, IPS Conf. Proc. 49, Bologna 1996, p. 825 - 841.

13. S. Hess, C. Aust, L. Bennett, M. Kröger, C. Pereira Borgmeyer, and T. WeIDER. Physica A 240 (1997) 126; S. HESS. Flow properties and structure of anisotropic fluids studied by Non-Equilibrium Molecular Dynamics and Flow properties of other complex fluids: polymeric liquids, ferro-fluids and magneto-rheological fluids, in: Advances in the computer simulation of liquid crystals, eds. P. Parisi and C. Zannoni (Kluwer, Dordrecht, 2000)

14. S. HESS AND T. WEIDER. Flow properties and structural changes in magneto-rheological fluids via non-equilibrium molecular dynamics, in: Proc. XIIth Int. Congr. Rheol., eds. A. Ait-Kadi, J.M. Dealy, D.F. James, and M.C. Williams (Can. Rheol. Group, Quebec, 1996) p. 313-314.

15. S.-Q. Wang, W.M. Gelbart and A. Ben-Shaul. J. Phys. Chem. 94 (1990) 2219.

16. M. Kröger and R. Makhloufi. Phys. Rev. E 53 (1996) 2531.

17. W. Carl, R. Makhloufi And M. Kröger. J. Phys. France II 7 (1997) 931.

18. M. KRöGER. Dynamics of actin filaments, in: Progress and Trends in Rheology V, ed. I. Emri (Steinkopff, Darmstadt, 1998) 338-39.

19. V.N. Tsvetkov. Acta. Physicochim. U.S.S.R. 10 (1939) 555.

20. H. Gasparoux and J. Prost. J. Phys. (Paris) 32 (1971) 953; J. Prost and H. Gasparoux. Phys. Lett. 36 A (1971) 245.

21. G. Heppke And F. Schneider. Z. Naturforsch. 27a (1972) 976; 28a (1973) 994.

22. J.P. McTague. J. Chem. Phys. 51 (1969) 133.

23. S. Odenbach. Int. J. Mod. Phys. B 14 (2000) 1615; Adv. Coll. Interf. Sci. 46 (1993) 263.

24. P.J. Rankin, J.M. Ginder, and D.J. Klingenberg. Curr. Opin. Coll. Interf. Sci. 3 (1998) 373. 
25. S. OdenBACh, AND H. StÖRK. J. magn. magn. mater. 183 (1998) 188; O. AMbacher, S. Odenbach, and K. Stierstadt. Z. Physik. B - Cond. Matter 86 (1992) 29.

26. S. Odenbach, T. Rylewicz, and M. Heyen. J. magn. magn. mater. 201 (1999) 155; S. Odenbach, T. Rylewicz, and H. Rath. Phys. Fluids 11 (1999) 10.

27. K.D. Weiss, J.D. Carlson, and D.A. Nixon. J. Intell. Mater. Syst. Struct. 5 (1994) 772.

28. S. Odenbach, H. Gilly, And P. Lindner. J. magn. magn. mater. 201 (1999) 353.

29. S. Odenbach. J. magn. magn. mater. 201 (1999) 149.

30. H.P. Gavin. J. Non-Newt. Fluid Mech. 71 (1997) 165.

31. W.G. Hoover. Molecular Dynamics, (Springer, Berlin, 1986); Computational Statistical Mechanics (Elsevier, Amsterdam, 1991)

32. W.T. Ashurst And W.G. Hoover. Am. Phys. Soc. 17 (1972) 1196; Phys. Rev. Lett. 31 (1972) 206; Phys. Rev. A11 (1975) 658.

33. E.M. Gosling, I.R. McDonald, And K. Singer. Mol. Phys. 26 (1973) 1475.

34. S. Hess And W. Loose. Physica A 162 (1989) 138.

35. W. Loose and S. Hess. Phys. Rev. Lett. 58 (1988) 2443; Phys. Rev. A 37 (1988) 2099.

36. G. Bossis, Y. Grasselli, E. Lemaire, A. Meunier, J.F. Brady, and T. Phung. Phys. Scripta T 49 (1993) 89.

37. S. Hess, J.B. Hayter, And R. Pynn. Mol. Phys. 53 (1984) 1527.

38. S. Hess, J. Schwarzl, And D. BaAlss. J. Phys. Condens. Matter 2 (1990) SA 279.

39. T. WEIDER. Simulation kristalliner Strukturen unter Scherung, Dissertation (Wissenschaft \& Technik Verlag, Berlin, 1995).

40. P. De Gennes and P. Pincus. Phys. Kondens. Mater. 11 (1970) 189.

41. M. KRöGer. Macromol. Chem. Macromol. Symp. 133 (1998) 101.

Received January 16, 2002 